Manuscript prepared as an article for Chemico-Biological Interactions

\title{
Lipases and Their Inhibitors in Health and Disease
}

\author{
Daniel K. Nomura ${ }^{1,2}$ and John E. Casida ${ }^{1,3}$
}




\begin{abstract}
Lipids play diverse and important biological roles including maintaining cellular integrity, storing fat for energy, acting as signaling molecules, and forming microdomains to support membrane protein signaling. Altering the levels of specific lipid species through activating or inactivating their biosynthetic or degradative pathways has been shown to provide either therapeutic benefit or cause disease. This review focuses on the functional, therapeutic, and (patho)physiological roles of lipases within the serine hydrolase superfamily and their inhibitors, with particular emphasis on the pharmacological tools, drugs, and environmental chemicals that inhibit these lipases.
\end{abstract}

Keywords: lipase, phospholipase, neutral lipases, lipase inhibitors 


\section{Introduction}

Lipases play critical roles in human health and disease. Beyond their importance in digestion, transport, and processing of dietary lipids, lipases function in hydrolyzing a variety of lipid substrates to modulate membrane integrity, lipid signaling, and lipid rafts. The substrates of lipases are diverse including neutral lipids, phospholipids and lysophospholipids, sphingolipids, ether lipids, oxidized lipids, and lipid protein modifications $^{1,2}$.

Specific lipases have been shown to be dysregulated or genetically linked to various human pathologies ${ }^{3,4}$. Genetic deletion or pharmacological inhibition of certain lipases has led to either therapeutic benefit or pathology in a variety of biological settings. Not surprisingly, some lipases have been targeted by pharmaceutical companies for therapeutic benefit and there are several drugs that are currently approved or are in clinical trials for obesity, pain, inflammation, anxiety, and cardiovascular disease ${ }^{5-7}$. Many other lipases and their inhibitors are effective in animal models, thus opening the possibility for further therapeutic exploitation of lipid hydrolyzing enzymes. However, inhibition of some lipases by environmental chemicals has also been shown to cause pathologies ranging from neurodegeneration to psychotropic effects to dyslipidemia ${ }^{8,9}$.

Most lipases belong to the serine hydrolase superfamily of enzymes that use the nucleophilic active-site serine to catalyze the hydrolysis of diverse lipid substrates ${ }^{2}$. The conserved biochemistry across this enzyme class, coupled with certain chemical scaffolds that target serine hydrolases, have enabled the development of inhibitors against many lipases. However, the reactivity of the serine nucleophile within lipase active-sites has also been a toxicological liability for various environmental electrophiles that covalently target the active-site serine. We review here the biochemical and physiological function of lipid hydrolases with particular emphasis on the pharmacological tools, drugs, and environmental chemicals that inhibit these lipases. We 
use the gene names for many of the lipases described here that have had multiple designations, and the substrates and products for most but not all of them are shown in

Figs. 1-3.

\section{Neutral Lipid Lipases}

\subsection{PNPLA2 or Adipose Triglyceride Lipase (ATGL)}

PNPLA2 or adipose triglyceride lipase (ATGL) is the rate-limiting step for triglyceride hydrolysis in adipose tissues with the highest expression in both white and brown adipose tissues (WAT/BAT), with lesser expression in skeletal and cardiac muscle and testes (Fig. 1A). PNPLA2(-/-) mice show $>80,44$, and $73 \%$ decrease in triacylglycerol hydrolytic activity in WAT, skeletal muscle, and liver, respectively, and show reduced release of fatty acids following isoproterenol stimulation. PNPLA2(-/-) mice show mild obesity with increased fat mass and larger WAT and BAT lipid droplets, and are intolerant to cold exposure or fasting, likely due to their lipolytic impairment and inability to release fatty acids for heat and energy production. These mice die prematurely from a large accumulation of triacylglycerols in the heart leading to cardiac dysfunction ${ }^{10}$.

Nonetheless, inhibiting ATGL may have potential therapeutic benefit. PNPLA2(-/) mice show improved glucose tolerance and insulin sensitivity under both normal and high-fat diet ${ }^{10-12}$. PNPLA2-deficient mice are also protected against adipose and muscle loss caused by cancer-associated cachexia ${ }^{13}$. Atglstatin is an ATGL inhibitor that has been reported to selectively inhibit triglyceride hydrolase activity in WAT and reduce free fatty acid release in vitro and in vivo in wild-type but not PNPLA2(-/-) mice, indicating the specificity of the inhibitor ${ }^{14}$ (Fig. 1B). Of future importance will be to understand whether pharmacological blockade of ATGL can provide therapeutic benefit without the cardiovascular toxicity. 


\subsection{LIPE or Hormone-Sensitive Lipase (HSL)}

LIPE or hormone-sensitive lipase (HSL) is a diacylglycerol and cholesteryl ester hydrolase that is expressed primarily in adipose tissue and adrenal glands with lower expression in cardiac and skeletal muscle, and macrophages (Fig. 1A) ${ }^{15,16}$. LIPE is regulated by phosphorylation involving protein kinase A signaling, which can be stimulated by catecholamines or suppressed by insulin, causing LIPE to be translocated from the cytosol to lipid droplets to activate its hydrolytic activity ${ }^{17,18}$. LIPE(-/-) mice show complete ablation of cholesteryl ester hydrolytic activity in WAT, BAT, and testes, but still possess $\sim 40 \%$ triacylglycerol hydrolytic activity in adipose tissue, consistent with the primary role of ATGL in adipose triacylglycerol hydrolysis ${ }^{19}$. These mice show adipocyte hypertrophy, but not obesity, and reduced isoproterenol-stimulated fatty acid release in WAT and accumulation of diacylglycerols in several tissues ${ }^{19,20}$. LIPE(-/-) mice are also infertile due to lack of spermatozoa ${ }^{19}$, are resistant to high-fat diet-induced obesity, show improved hepatic insulin sensitivity, and are protected from short-term diet-induced insulin resistance in skeletal muscle and heart ${ }^{21,22}$.

Inhibitors of HSL include heterocyclic ureas and carbamates such as Ebdrup $13 f$ from Novo Nordisk and BAY from Bayer Healthcare, but their selectivity remains unknown (Fig. 1B) ${ }^{23,24}$. BAY has been shown to inhibit forskolin-activated lipolysis both in vitro and ex vivo, lower plasma free fatty acid and glycerol levels in vivo in overnightfasted mice and dogs, and reduce hyperglycemia in streptozotocin-induced diabetic rats ${ }^{23}$. Ebdrup $13 \mathrm{f}$ has been shown to reduce lipolysis in vivo in fasted rats ${ }^{24}$.

\subsection{Triglyceride Hydrolase or Carboxylesterase 3 (Ces3)}

Endoplasmic reticulum-localized triacylglycerol hydrolase or carboxylesterase 3 (Ces3) (in mice) or CES1 (in humans) has been suggested to act as the liver triacylglycerol 
hydrolase involved in the lipolysis of triacylglycerol-containing lipid droplets for the eventual re-esterification and packaging into very low-density lipoproteins (VLDLs) for triacylglycerol delivery to peripheral tissues (Fig. 1A). Consistent with this role, Ces3(-/-) mice show reduced plasma triacylglycerol, apolipoprotein $B$, and fatty acid levels in both fasted and fed states ${ }^{25}$. Interestingly, these mice do not possess increased hepatic triacylglycerol levels due to an increase in respiratory quotient and energy expenditure ${ }^{25}$. Ces3 activity is also markedly increased during adipocyte differentiation.

Several CES3 inhibitors have been described including GR148672X and WWL113 (Fig. 1B). GR148672X decreases secretion of triacylglycerols, cholesteryl esters, phosphatidylcholine, and ApoB100 from primary rat hepatocytes ${ }^{26}$. WWL113 promotes lipid storage in adipocytes and ameliorates weight increase, serum glucose, fatty acids, triacylglycerols, and glucose intolerance in a diet-induced and $d b / d b$ mouse model of metabolic syndrome ${ }^{27}$.

\subsection{Secreted Triglyceride Lipases: Pancreatic Lipase (PNLIP), Carboxylester Lipase (CEL), and Gastric Lipase (LIPF)}

PNLIP, CEL, and LIPF are secreted into or attached to the intestinal lumen for digestion of dietary fat ${ }^{28}$. These three enzymes all hydrolyze triacylglycerols and diacylglycerols to monoacylglycerols and fatty acids ${ }^{29-34}$ (Fig. 1A). CEL has broader substrate specificity than PNLIP and LIPF and also hydrolyzes monoacylglycerols, phospholipids, lysophospholipids, and ceramides ${ }^{29-34}$. PNLIP is expressed in the pancreas and is secreted directly into the intestinal lumen ${ }^{35}$. CEL is primarily expressed in pancreas and mammary glands, and is secreted into pancreatic juice and milk and is attached to intestinal cells ${ }^{36-38}$. LIPF is secreted by gastric mucosal cells ${ }^{39}$. CEL, but not PNLIP and LIPF, requires bile salts for activity ${ }^{40}$. PNLIP(-/-) mice have delayed triacylglycerol and 
cholesterol absorption ${ }^{41}$. CEL(-/-) mice show accumulation of intestinal lipid droplets and intestinal damage in pups ${ }^{42}$. LIPF(-/-) mice have not yet been generated.

While there are no selective inhibitors that specifically inhibit these enzymes, tetrahydrolipstatin (THL) or Orlistat is a non-specific lipase inhibitor that inhibits all three enzymes as well as other lipases (Fig. 1B). Orlistat is an approved drug for treatment of obesity through preventing the absorption of dietary fat and reducing caloric intake, albeit this drug may cause steatorrhea ${ }^{7}$.

\subsection{Hepatic Lipase (LIPC)}

LIPC is secreted by the liver and localized at the luminal surface of hepatic endothelial cells ${ }^{43}$. LIPC hydrolyzes both triacylglycerols and phospholipids on lipoproteins (Fig. 1A) ${ }^{44,45}$. However, LIPC(-/-) mice show increased basal plasma cholesterol and phospholipids but not triacylglycerol ${ }^{46}$. There are no reported LIPC inhibitors.

\subsection{Endothelial Lipase (LIPG)}

LIPG is a lipid hydrolase synthesized by endothelial cells in vitro and expressed in vivo in organs including liver, lung, kidney, and placenta, but not in skeletal muscle. LIPG has substantial phospholipase activity but less triglyceride hydrolase activity. Overexpression of LIPG in mice reduces plasma HDL-cholesterol and protein apolipoprotein A-I concentrations ${ }^{47}$. Inhibition in mice or loss of function variants in humans of LIPG results in reduced HDL-cholesterol levels, suggesting an important role of LIPG in HDL metabolism in vivo ${ }^{48,49}$. Because of its potential for therapeutic action towards cardiovascular disease, several LIPG inhibitors have been synthesized, including the anthranilic acid XEN445 which has been shown to also elevate plasma HDL-cholesterol levels in mice (Fig. 1B) ${ }^{50}$. 


\subsection{Lipoprotein Lipase (LPL)}

LPL is anchored on capillary endothelial cells through heparin sulfate-proteoglycans where it hydrolyzes triacylglycerols in mature chylomicrons and VLDL particles, upon docking with apolipoprotein C-II, to fatty acids and monoglycerides for import of fatty acids into peripheral organs (Fig. 1A). LPL deficiency in both rodents and humans causes hugely elevated serum triglyceride levels and VLDL and chylomicron levels ${ }^{51,52}$. LPL(-/-) mouse pups die within $18 \mathrm{~h}$ of suckling due to hyper-elevated serum triglyceride levels ${ }^{52}$. There are several urea-based LPL inhibitors such as GSK264220A that also inhibit LIPG (Fig. 1B) ${ }^{53}$.

\subsection{Lysosomal Acid Lipase (LIPA)}

LIPA is localized in early and late endosomal membranes and has optimal activity in acidic $\mathrm{pH}^{54}$. It hydrolyzes cholesteryl esters, triacylglycerols, diacylglycerols, and monoacylglycerols (Fig. 1A) ${ }^{55}$. LIPA supplies cells with cholesterol from internalized LDL particles ${ }^{56}$. LIPA(-/-) mice show dramatic elevations in triacylglycerol and cholesteryl ester levels in liver, adrenal glands, and small intestines ${ }^{57,58}$. LIPA-deficiency in humans causes Wolman or cholesteryl ester storage disease (CESD) ${ }^{59-61}$. Wolman disease is associated with complete loss of LIPA activity leading to lysosomal triacylglycerol accumulation, vomiting, diarrhea, and hepatosplenomegaly, with most patients dying within the first year of their lives. CESD patients show residual LIPA activity and show hypercholesterolemia and survive into adulthood. Several inhibitors for LIPA have been described, although their selectivity has not been confirmed ${ }^{62}$.

\subsection{DDHD2}

DDHD2 is the primary brain triglyceride hydrolase (Fig. 1A). Loss of function mutations in DDHD2 leads to a genetic disorder termed complex hereditary spastic paraplegia 
characterized by spasticity in lower limbs due to nerve damage. Metabolomic profiling of DDHD2(-/-) mice revealed massive elevations in triacylglycerol levels in the brain, but not in peripheral tissues. Large lipid droplets accumulate in DDHD2(-/-) neuronal cell bodies accompanied by impairments in motor and cognitive function ${ }^{3}$. KLH45 is a selective triazole urea irreversible inhibitor of DDHD2 that also leads to brain triacylglycerol accumulation ${ }^{3}$. While DDHD2 inhibition is not advisable as a therapeutic, $\mathrm{KLH} 45$ is a promising tool compound for investigating the role of triglyceride metabolism in the brain and how it drives hereditary spastic paraplegia (Fig. 1B).

\subsection{Monoacylglycerol Lipase (MAGL)}

Monoacylglycerol lipase (MAGL) is the primary hydrolytic enzyme for monoacylglycerols in most tissues, including brain, liver, kidney, spleen, heart, WAT, BAT, and lung (Fig. 1A) ${ }^{63}$. One of the monoacylglycerols, 2-arachidonoylglycerol (2-AG), is an endogenous cannabinoid signaling lipid that acts as an agonist on cannabinoid receptors CB1 and CB2 (described later) ${ }^{64,65}$.

Several highly selective irreversible carbamate MAGL inhibitors have been developed, including JZL184, KML29, and JW651 (Fig. 1B) ${ }^{66-68}$. Both MAGL(-/-) mice and mice treated with JZL184 show 4-10-fold elevations in brain levels of 2-AG ${ }^{66,69-71}$. MAGL blockade produces tissue-specific differences in monoglyceride metabolism with brain showing the most dramatic elevations in 2-AG and peripheral tissues showing greater changes in other monoglycerides, consistent with the role of MAGL as the final lipolytic step in triacylglycerol breakdown ${ }^{70}$. Genetic and pharmacological ablation of MAGL also lowers arachidonic acid and pro-inflammatory prostaglandins in specific tissues under both basal conditions and lipopolysaccharide (LPS)-induction, including in brain, liver, and lung, indicating that MAGL provides the major arachidonic acid source for prostaglandin synthesis in these tissues ${ }^{69}$. 
Acute pharmacological blockade of MAGL in mice exerts CB1-dependent antinociceptive, anxiolytic, and anti-inflammatory effects and shows protection in inflammatory bowel disease models, without cognitive impairments or "high" associated with direct cannabinoid receptor agonists ${ }^{64,65}$. Both genetic and pharmacological ablation of MAGL also: 1) reduces LPS-induced neuroinflammation ${ }^{69}$;2) protects against neuroinflammation and neurodegeneration in Parkinson's disease mouse models ${ }^{69,72}$; 3) reduces inflammation, amyloid- $\beta$ plaques, and neurodegeneration, and improves integrity of hippocampal synaptic structure and function, spatial learning, and memory in Alzheimer's disease mouse models ${ }^{73,74}$; and 4 ) reduces fever ${ }^{75}$. These neuroprotective and antipyretic effects of MAGL inhibition are independent of cannabinoid receptor signaling indicating that they are likely caused by reduced proinflammatory eicosanoids. Genetic and pharmacological MAGL inactivation has also been shown to exert hepatoprotective effects in an ischemia-reperfusion injury and carbon tetrachloride-induced liver fibrosis model in mice through heightened CB2 receptor signaling and reduced eicosanoid levels ${ }^{76}$.

Interestingly, MAGL(-/-) mice and mice chronically treated with a MAGL inhibitor at a dose that causes full MAGL blockade loose CB1-mediated antinociceptive effects and show cross-tolerance to $\mathrm{CB} 1$ receptor agonists, display physical dependence due to functional antagonism of the $\mathrm{CB} 1$ receptor, and show impaired CB1 dependent synaptic plasticity ${ }^{71}$. These effects result from prolonged and heightened 2-AG levels and CB1 stimulation causing CB1 receptor desensitization and functional antagonism ${ }^{71}$. MAGL(-/) mice also exhibit reduced plasma glycerol and triacylglycerol as well as liver triacylglycerol levels under fasted conditions, indicating impaired lipolysis. Under high-fat diet, MAGL(-/-) mice also show significantly improved glucose tolerance and insulin sensitivity, despite equal weight gain as wild-type counterparts ${ }^{77}$. 
Based on studies using neuronal and astrocyte-specific MAGL(-/-) mice, both neurons and astrocytes coordinately regulate $2-A G$ content and endocannabinoiddependent forms of synaptic plasticity and behavior. Astrocytic MAGL is mainly responsible for converting 2-AG to prostaglandins that may involve transcellular transport of lipid substrates ${ }^{78}$. MAGL has also been shown to be upregulated in aggressive human cancers and MAGL inactivation impairs cancer cell motility and tumor xenograft growth in mice due to reduced levels of fatty acids and fatty acid-derived protumorigenic signaling lipids such as lysophosphatidic acid ${ }^{79,80}$. Due to the various therapeutic indications, MAGL inhibitors have garnered interest for clinical development. MAGL inhibitors are currently in Phase I clinical trials ${ }^{81}$.

\subsection{Diacylglycerol Lipase (DAGL)}

DAGL $\alpha$ and DAGL $\beta$ are diacylglycerol-hydrolyzing enzymes and the primary biosynthesizing enzyme for the endocannabinoid signaling lipid 2-arachidonoylglycerol (2-AG) (Fig. 1A) ${ }^{82-84}$. DAGL $\alpha$ and DAGL $\beta$ prefer hydrolysis of the $s n-1$ position of diacylglycerols compared to $s n-2^{82}$. DAGL $\alpha(-/-)$ mice show an $~ 80 \%$ reduction in the brain and spinal cord and $\sim 50 \%$ reduction in adipose and liver in 2-AG levels, and $\sim 75$ $80 \%$ reduction in brain and spinal cord and $\sim 60 \%$ reduction in liver and no reduction in adipose tissue in arachidonic acid levels. DAGL $\beta(-/-)$ mice show $\sim 50 \%$ reduction in brain, $90 \%$ reduction in liver, and no change in spinal cord or adipose tissue in 2-AG levels. Arachidonic acid levels are $25 \%$ lower in brain, $75 \%$ lower in liver, and no change in spinal cord or adipose tissue in DAGL $\beta(-/-)$ mice ${ }^{83}$. Retrograde endocannabinoid 2-AG signaling at the CB1 receptor in the hippocampus can reduce GABA release from presynpases causing depolarization-induced suppression of inhibition (DSI). DAGL $\alpha(-/-)$ mice show no DSI, consistent with the loss of 2-AG levels in 
the brain. Both DAGL $\alpha(-/-)$ and DAGL $\beta(-/-)$ mice also have reduced neurogenesis in the brain ${ }^{83,84}$. DAGL $\alpha(-/-)$ mice show reduced exploration of central area of the open field, a maternal neglect behavior, a fear extinction deficit, increased behavioral despair, and increased anxiety-related behaviors, indicating that loss of DAGL $\alpha$ activity results in enhanced anxiety, stress, and fear responses ${ }^{85}$. DAGL $\beta(-/-)$ mice also show lower 2AG, arachidonic acid, and pro-inflammatory eicosanoids in mouse peritoneal macrophages leading to a reduction in LPS-induced TNF $\alpha$ release ${ }^{86}$.

Recent DAGL inhibitors include the DAGL $\beta$-selective KT109 and KT172 and the dual DAGL $\alpha / \beta$ inhibitor LEI105 (Fig. 1B) ${ }^{86,87}$. KT109 and KT172 treatment in vivo in mice leads to reduced 2-AG, arachidonic acid, and pro-inflammatory prostaglandin levels and LPS-induced TNF $\alpha$ release in peritoneal macrophages. LEl105 treatment in Neuro2A cells reduced 2-AG levels and CB1-mediated short-term synaptic plasticity.

\subsection{KIAA1363}

KIAA1363 is the primary enzyme responsible for 2-acetyl monoalkylglycerol ether (2acetyl MAGE) hydrolysis in brain, lung, heart, and kidney (Fig. 1A). 2-Acetyl MAGE is the penultimate precursor to generating de novo pools of platelet activating factor (PAF) 88,89. In vitro in brain membrane proteomes, 2-acetyl MAGE is diverted to generating more PAF in KIAA1363(-/-) mice with a reduction in MAGE production, but this metabolic effect is not evident in endogenous levels of PAF or MAGE ${ }^{90}$. KIAA1363 is also highly upregulated in aggressive human cancer cells and inactivation of this enzyme impairs cancer cell motility and in vivo tumor xenograft growth in mice ${ }^{79,89}$. Metabolomic profiling revealed that KIAA1363 inactivation lowers the levels of MAGE as well as the downstream pro-tumorigenic signaling lipid alkyl lysophosphatidic acid ${ }^{79,80}$. The true physiological role of this enzyme still remains poorly understood. 
Several selective irreversible carbamate inhibitors of KIAA1363 have been developed including AS115 and JW480 (Fig. 1B) ${ }^{89,91}$. JW480 treatment lowers MAGE levels and impairs motility and tumor growth in prostate cancer cells ${ }^{91}$. KIAA1363 inhibition by JW480 blocks platelet aggregation, megakaryocyte function, and thrombus formation ${ }^{92}$. KIAA1363 is also the primary detoxification enzyme in the brain for the bioactivated insecticide metabolite chlorpyrifos oxon ${ }^{93,90}$, and KIAA1363(-/-) mice show increased sensitivity to the pesticide chlorpyrifos ${ }^{90}$.

\section{Amidases: Fatty Acid Amide Hydrolase (FAAH)}

FAAH is the primary hydrolytic enzyme for $\mathrm{N}$-acylethanolamines, $\mathrm{N}$-acylamides, and $\mathrm{N}$ acyltaurines (Fig. 2A) ${ }^{94,95}$. FAAH is highly expressed in brain, liver, kidney, and testes, but not heart or skeletal muscle. Related bioactive lipids include the endocannabinoid signaling lipid $N$-arachidonoylethanolamine (anandamide), the sleep-inducing factor oleamide, and the transient receptor potential ion channel agonists $N$-acyltaurines ${ }^{95}$. The biosynthetic pathway for anandamide is shown in Fig. 2A.

FAAH(-/-) mice show $>10$-fold elevations in brain anandamide levels. $N$ Acylethanolamines are elevated most dramatically in brain and liver, but are also elevated in plasma, testis, heart, lung, and kidney of $\mathrm{FAAH}(-/-)$ and $\mathrm{FAAH}$ inhibitortreated mice. $\mathrm{N}$-Acyltaurines are elevated in plasma, BAT, heart, spleen, lung, testis, kidney, and most dramatically in liver ${ }^{96-100}$. $\mathrm{FAAH}(-/-)$ mice as well as mice treated with FAAH inhibitors show cannabinoid-dependent anxiolytic, antidepressive, and antinociceptive effects without the cognitive side-effects associated with direct CB1 agonists ${ }^{98,101-107}$. Because of the therapeutic potential of FAAH inhibition, many highly selective and in vivo efficacious FAAH inhibitors have been developed including earlier tool compounds such as the carbamate URB597 and $\alpha$-ketoheterocycle OL-135 and 
later more advanced compounds such as the piperidine urea PF-3845 and the clinical candidate PF-04457845 (Fig. 2B) ${ }^{99-101,108}$. Treatment with these inhibitors elevates anandamide levels and exerts antinociceptive and anti-inflammatory effects in various pain and inflammatory mouse models. FAAH inhibitors are now in various stages of clinical trials for pain and other modalities ${ }^{109}$.

\section{Phospholipases}

\subsection{General Phospholipase Activity}

Phospholipids are cleaved at any one of four positions by phospholipases PLA1, PLA2, PLC, and PLD (Fig. 3A). There are many PLA1 and PLA2 enzymes in the serine hydrolase family, and we will focus attention on those that have been the bestcharacterized and have pharmacological tools.

\subsection{Phospholipase A2 (PLA2G4A-F)}

PLA2 enzymes hydrolyze the sn-2 acyl chain from phospholipids to produce a fatty acid and a lysophospholipid (Fig. 3A). The sn-2 acyl chain can be short (e.g. acetyl), medium chain (e.g. butyryl), long chain (palmitoyl, stearoyl), monounsaturated (e.g. oleoyl), polyunsaturated (e.g. arachidonoyl), or oxidatively truncated (e.g. azaeaoyl, oxovaleryl) 2 .

The PLA2G4A subfamily of phospholipases include the cytosolic PLA2s PLA2G4A-F, of which PLA2G4B-F are less characterized ${ }^{110}$. PLA2G4A, also known as cPLA2, is expressed in nearly every tissue with highest expression in immune cells and hydrolyzes phosphatidylcholine (PC) and phosphatidylethanolamine (PE) in a calciumdependent manner ${ }^{111}$. cPLA2 prefers arachidonoyl as the sn-2 position and is thought to provide the primary arachidonic acid source for generating eicosanoids ${ }^{110}$. PLA2G4A(-/) mice show lower eicosanoid production in peritoneal macrophages under both basal 
and LPS-stimulated conditions ${ }^{112}$. PLA2G4A(-/-) mice show neuroprotective effects in stroke mouse models and have reduced anaphylactic responses in allergic models, hepatoprotective effects in fibrosis models, and resistance against experimental autoimmune encephalomyelitis ${ }^{110,112-115}$. While PLA2G4A has been considered to be the dominant source of arachidonic acid for eicosanoid production, recent studies indicate that other hydrolytic enzymes such as DAGL and MAGL may play this role in certain tissue and cellular contexts ${ }^{69,116}$. Because PLA2G4A can control eicosanoid production, much like the anti-inflammatory cyclooxygenase inhibitors, there has been significant interest in developing PLA2G4A inhibitors as anti-inflammatory agents ${ }^{110}$. Several PLA2G4A inhibitors have been developed, including pyrrophenone and Ecopladib that show selectivity over other PLA2 enzymes (Fig. 3B) ${ }^{117,118}$. Ecopladib has shown efficacy in inflammatory rodent models such as the rat carrageenan air pouch and induced paw edema models ${ }^{118}$.

\subsection{PLA2G6}

PLA2G6 is a calcium-independent PLA2 or IPLA2 with broad tissue expression that hydrolyzes broad range of substrates including PCs, PAF, and lysophospholipids ${ }^{119,120}$. PLA2G6(-/-) mice show significantly reduced PC hydrolase activity in brain, muscle, kidney, liver, and epididymis, but do not show changes in PC levels ${ }^{121,122}$. PLA2G6(-/-) mice show reduced fertility due to impaired spermatozoa and age-related neurological deficits including gait problems and clasping behaviors with peripheral demyelination and accumulation of spheroid bodies in axons and synapses ${ }^{121,122}$. Mutations in the PLA2G6 gene in humans leads to infantile neuroaxonal dystrophy, a fatal disease characterized by cerebellar ataxia, hypotonia of the trunk, spastic quadriplegia, and hyperreflexia caused by axonal swelling and accumulation of spheroid bodies in the nervous system ${ }^{123,124}$. The endogenous substrates of PLA2G6 and the mechanisms 
underlying these neurological conditions remain unknown and there are currently no PLA2G6 inhibitors.

\subsection{PLA2G7}

PLA2G7, also called lipoprotein-associated PLA2, is secreted by monocytes and found in the plasma associated with both LDL and HDL particles and is expressed in the brain 125-127. PLA2G7 hydrolyzes the sn-2 acetyl group from PAF or oxidatively truncated sn-2 acyl chains on PCs such as az-PAF in vitro, with no activity on medium to long-chain acyl chains (Fig. 3A) ${ }^{125,128,129}$. This lipase generates pro-inflammatory lysophosphatidylcholine (LPC) and fatty acids as products. PLA2G7 has been implicated in many pathologies, including atherosclerosis, inflammation, and Alzheimer's disease 5,130. Many PLA2G7 inhibitors have been developed including two clinical candidates Darapladib and Rilapladib and the carbamate JMN21 (Fig. 3B) ${ }^{131-133}$. Darapladib treatment results in reduced development of advanced coronary atherosclerosis in diabetic and hypercholesterolemic swine. Darapladib markedly inhibited plasma and lesion PLA2G7 activity and reduced lesion LPC content ${ }^{134}$. Darapladib treatment in pigs also reduced the extent of immunoglobulin $\mathrm{G}$ brain parenchyma penetration suggesting a reduction in blood brain barrier leakage and significantly lowered amyloid $\beta_{1-42}$ deposition, indicating that PLA2G7 inhibitors may be effective in treating Alzheimer's disease ${ }^{135}$. Several clinical trials with PLA2G7 inhibitors have been completed but have unfortunately not shown any benefit in protecting against cardiovascular death, myocardial infarction, or stroke ${ }^{5}$.

\subsection{PNPLA6}

PNPLA6, also called neuropathy target esterase (NTE), is expressed in the brain and testes, and is a target for organophosphorus-induced delayed neuropathy that causes 
axonal degradation and onset of ataxia and lower limb paralysis in 1-3 weeks after initial exposure ${ }^{9,136,137}$. While PNPLA6(-/-) mice have midgestational lethality, brain-specific PNPLA6-deficiency in mice results in disruption of the endoplasmic reticulum, vaculation of nerve cell bodies, and abnormal reticular aggregates ${ }^{137}$. PNPLA6 has been shown to hydrolyze phospholipids, lysophospholipids (lysophosphatidylcholine is often used for assays as shown later), and monoglycerides in vitro, but the physiological substrate of PNPLA6 is still not understood ${ }^{138,139}$.

\subsection{Platelet Activating Factor Acetylhydrolase 2 (PAFAH2)}

PAFAH2 hydrolyzes PAF and oxidatively truncated PCs (Fig. 3A) ${ }^{140,141}$. PAFAH2(-/-) mice show near complete reductions in PAF hydrolytic activity in kidney and liver, but not brain or plasma ${ }^{142}$. PAFAH2(-/-) mice are more sensitive to carbon tetrachloride-induced liver injury and mouse embryonic fibroblasts from PAFAH2(-/-) mice are more sensitive to peroxide-induced cell death, indicating that PAFAH2 is important in detoxifying oxidized phospholipids ${ }^{142}$. AA39-2 is a highly selective triazole urea irreversible inhibitor of PAFAH2 (Fig. 3B) ${ }^{143}$.

\subsection{PAFAH1B2 and PAFAH1B3}

PAFAH1B2 and PAFAH1B3 are part of a trimeric complex that includes a noncatalytic and non-serine hydrolase PAFAH1B1 subunit, also called LIS1 ${ }^{144,145}$. The PAFAH1B complex hydrolyzes PAF and oxidized PCs, but not PC, PE, or LPC, but the physiological substrates of these enzymes remain unknown ${ }^{141}$. In mice, PAFAH1B1 and PAFAH1B2 are expressed in nearly every tissue with highest levels in brain, lung, and testis whereas PAFAH1B3 expression is restricted to brain and testis ${ }^{146}$. PAFAH1B1(-/-) mice develop type I lissencephaly, a cephalic disorder characterized by lack of brain fold and groove development and is embryonically lethal ${ }^{147}$. PAFAH1B2(-/-) or 
PAFAH1B2/PAFAH1B3(-/-) mice are viable and overtly normal but show lower testes weight and impaired spermatogenesis, leading to infertility in males ${ }^{146}$. PAFAH1B3 is heightened in human cancers and correlated with poor prognosis ${ }^{148,149}$. Knockdown of PAFAH1B2 or PAFAH1B3 impairs breast cancer cell survival, proliferation, and in vivo tumor growth and causes wide-spread alterations in cancer cell lipid metabolism ${ }^{149}$. A high throughput screen yielded a highly selective and reversible PAFAH1B2 and PAFAH1B3 dual tetrahydropyridine inhibitor P11, which also impairs cell survival ${ }^{149,150}$.

\subsection{Alpha/beta hydrolase domain-containing protein 3 (ABHD3)}

ABHD3 is the primary enzyme for hydrolyzing medium-chain and oxidatively truncated phospholipids (Fig. 3A). ABHD3(-/-) mice are normal and viable and possess heightened levels of multiple medium-chain PCs and LPCs. No inhibitors exist for ABHD3 and the therapeutic or pathological effects of inhibiting ABHD3 are not yet known.

\subsection{ABHD4}

ABHD4 deacylates $\mathrm{O}$-acyl chains from $\mathrm{N}$-acylphosphatidylethanolamines (NAPEs) as well as $\mathrm{N}$-acylphosphatidylserines. ABHD4 acts on both NAPEs and lysoNAPEs but does not act on other lysophospholipids. NAPEs are thought to be the precursor for generating $\mathrm{N}$-acylethanolamines as well as the endocannabinoid anandamide. ABHD4(-

/-) mice show reductions in brain glycerophospho- $N$-acylethanolamine and plasmalogenbased lyso-NAPEs, as well as $\mathrm{N}$-acyl lysophosphatidylserines ${ }^{151}$. There are no ABHD4 inhibitors yet reported.

\subsection{ABHD16A and ABHD12}


ABHD16A and ABHD12 hydrolyze phosphatidylserine and lysophosphatidylserine, respectively (Fig. 3A) ${ }^{4,152}$. Mutations in ABHD12 in humans cause a neurodegenerative disorder PHARC (polyneuropathy, hearing loss, ataxia, retinosis pigmentosa, and cataract). ABHD12(-/-) mice display massive increases in a rare set of pro-inflammatory very long chain lysophosphatidylserine lipids that stimulate toll-like receptor 2 , leading to age-dependent microglial activation and auditory and motor defects that resemble PHARC. Lysophosphatidylserine hydrolytic activity is substantially reduced in ABHD12(/-) mouse brains ${ }^{4}$. ABHD16A is the primary brain phosphatidylserine hydrolase that generates the lysophosphatidylserine which ABHD12 further hydrolyzes. ABHD16A(-/-) mice show decreased lysophosphatidylserine in brain and macrophages and reduces LPS-induced cytokine production in macrophages. A selective ABHD16A inhibitor KC01 has been reported that lowers lysophosphatidylserine secretion and LPS-induced cytokine release from macrophages and restores the elevated lysophosphatidylserine and cytokine levels in ABHD12(-/-) mice (Fig. 3B) ${ }^{152}$.

\section{Environmental Agents that Inhibit Lipases}

Understanding how environmental chemicals interact with protein targets, including lipases, is critical in evaluating agents that may potentiate or cause human disease. Several pesticides, plasticizers, and flame retardant chemicals covalently and irreversibly inhibit the serine nucleophile of multiple lipases ${ }^{8,153-156}$. This includes organophosphorus and thiocarbamate insecticides, herbicides, and fungicides, organophosphorus flame retardants, and organophosphorus plasticizers (Fig. 4).

In the 1930s during the Prohibition era, Jamaica Ginger, which contained tri-ocresylphosphate (TOCP), was consumed in alcoholic beverages leading to inhibition of NTE or PNPLA6, resulting in organophosphate-induced delayed neuropathy (described under PNPLA6) ${ }^{9}$. TOCP is bioactivated to 2 -(o-cresyl)-4- $H-1,2,3-$ 
benzodioxaphosphoran-2-one, a potent inhibitor of NTE ${ }^{157}$. Localized accumulation of lysophosphatidylcholine may then lead to demyelination and neuropathy ${ }^{139}$ (Fig. 4A). Aging of NTE following phosphorylation is an essential step in the delayed neuropathy but the mechanism remains unclear.

The organophosphorus insecticide chlorpyrifos inhibits many lipases, i.e. KIAA1363, MAGL, FAAH, ABHD6, HSL, and ABHD3 in the brain and CES1, CES1B, CES1F, CES2, CES2B, CES2G, CES3, CES5, CES6, MAGL, FAAH, and AADAC in liver, in vivo in mice, among other serine hydrolases ${ }^{8,154,155}$. MAGL and FAAH represent particularly common in vivo off-targets of organophosphorus and thiocarbamates pesticides (Fig. 4B). Chlorpyrifos, profenofos, tribufos, and molinate inhibit both MAGL and $\mathrm{FAAH}$, raise brain levels of 2-AG and anandamide, and causes cannabinoid-like behaviors ${ }^{8}$. While selective inhibition of MAGL or FAAH produce antinociceptive effects without psychotropic effects, dual inhibition of MAGL and FAAH inhibitor causes cannabinoid-mediated cataleptic behaviors ${ }^{158}$. The organophosphorus flame retardant triphenyl phosphate inhibits Ces1g in mice leading to increased hepatic diacylglycerol levels and protein kinase C stimulation and to serum hypertriglyceridemia ${ }^{156}$.

\section{Conclusions}

Lipases play critical functions in lipid biochemistry and regulate nearly every aspect of physiology. Lipase inhibitors have therefore been developed as both tool compounds as well as clinical candidates for treatment of inflammation, cardiovascular disease, and pain. These include non-specific gastric and pancreatic lipase inhibitors like Orlistat for obesity, MAGL and FAAH inhibitors for pain and inflammation, PLA2G4A inhibitors for inflammation, and PLA2G7 inhibitors for cardiovascular diseases ${ }^{2}$. There have also been selective and in vivo efficacious inhibitors developed against other lipases that may prove to demonstrate therapeutic benefit, including DAGL or ABHD16A inhibitors for 
inflammation, CES3, ATGL, and LIPE inhibitors for type II diabetes, or KIAA1363 and PAFAH1B2/1B3 inhibitors for cancer. These inhibitors are likely just the tip of the iceberg in terms of the therapeutic potential for targeting the many other lipases that are not mentioned here in this review. On the other hand, inhibiting certain lipases may cause debilitating pathologies such as delayed neuropathy with NTE, spastic paraplegia with DDHD2, and PHARC with ABHD12. Thus, it is also of importance to understand how environmental chemicals may be causing toxicity through inhibiting lipases.

Tapping deeper into targeting the lipase enzyme class is currently limited by our lack of understanding of (patho)physiological or therapeutic functions of the many yet uncharacterized lipases. Understanding their function and developing pharmacological and genetic tools for inhibiting these lipases will undoubtedly provide novel insights into disease progression as well as therapeutic targets.

\section{Acknowledgements}

Preparation of this manuscript was supported by the following grants to DKN: National Institutes of Health (P42ES004705; R01CA172667), American Cancer Society Research Scholar Award (RSG-14-242-01-TBE), DOD Breakthroughs Award (CDMRP W81XWH15-1-0050), and the BASF-CARA Award. The research of JEC was funded by the National Institutes of Health (P01ES00049) and the University of California at Berkeley. This report was presented by JEC at the $12^{\text {th }}$ Cholinesterases $-6^{\text {th }}$ Paraoxonase Meeting at Iche, Spain, in September 2015. 


\section{References}

1. Wymann, M. P. \& Schneiter, R. Lipid signalling in disease. Nat. Rev. Mol. Cell Biol. 9, 162-176 (2008).

2. Long, J. Z. \& Cravatt, B. F. The metabolic serine hydrolases and their functions in mammalian physiology and disease. Chem. Rev. 111, 6022-6063 (2011).

3. Inloes, J. M. et al. The hereditary spastic paraplegia-related enzyme DDHD2 is a principal brain triglyceride lipase. Proc. Natl. Acad. Sci. U. S. A. 111, 14924-14929 (2014).

4. Blankman, J. L., Long, J. Z., Trauger, S. A., Siuzdak, G. \& Cravatt, B. F. ABHD12 controls brain lysophosphatidylserine pathways that are deregulated in a murine model of the neurodegenerative disease PHARC. Proc. Natl. Acad. Sci. U. S. A. 110, 1500-1505 (2013).

5. Karabina, S. \& Ninio, E. Plasma PAFAH/PLA2G7 Genetic Variability, Cardiovascular Disease, and Clinical Trials. The Enzymes 38, 145-155 (2015).

6. Ahn, K. et al. Mechanistic and pharmacological characterization of PF-04457845: a highly potent and selective fatty acid amide hydrolase inhibitor that reduces inflammatory and noninflammatory pain. J. Pharmacol. Exp. Ther. 338, 114-124 (2011).

7. Kim, G. W., Lin, J. E., Blomain, E. S. \& Waldman, S. A. Antiobesity pharmacotherapy: new drugs and emerging targets. Clin. Pharmacol. Ther. 95, 53-66 (2014).

8. Nomura, D. K. \& Casida, J. E. Activity-based protein profiling of organophosphorus and thiocarbamate pesticides reveals multiple serine hydrolase targets in mouse brain. J. Agric. Food Chem. 59, 2808-2815 (2011).

9. Glynn, P. A mechanism for organophosphate-induced delayed neuropathy. Toxicol. Lett. 162, 94-97 (2006). 
10. Haemmerle, G. et al. Defective lipolysis and altered energy metabolism in mice lacking adipose triglyceride lipase. Science 312, 734-737 (2006).

11. Kienesberger, P. C. et al. Adipose triglyceride lipase deficiency causes tissuespecific changes in insulin signaling. J. Biol. Chem. 284, 30218-30229 (2009).

12. Hoy, A. J. et al. Adipose triglyceride lipase-null mice are resistant to high-fat dietinduced insulin resistance despite reduced energy expenditure and ectopic lipid accumulation. Endocrinology 152, 48-58 (2011).

13. Das, S. K. et al. Adipose triglyceride lipase contributes to cancer-associated cachexia. Science 333, 233-238 (2011).

14. Mayer, N. et al. Development of small-molecule inhibitors targeting adipose triglyceride lipase. Nat. Chem. Biol. 9, 785-787 (2013).

15. Reue, K., Cohen, R. D. \& Schotz, M. C. Evidence for hormone-sensitive lipase mRNA expression in human monocyte/macrophages. Arterioscler. Thromb. Vasc. Biol. 17, 3428-3432 (1997).

16. Holm, C. et al. Hormone-sensitive lipase: sequence, expression, and chromosomal localization to 19 cent-q13.3. Science 241, 1503-1506 (1988).

17. Egan, J. J. et al. Mechanism of hormone-stimulated lipolysis in adipocytes: translocation of hormone-sensitive lipase to the lipid storage droplet. Proc. Natl. Acad. Sci. U. S. A. 89, 8537-8541 (1992).

18. Kraemer, F. B. \& Shen, W.-J. Hormone-sensitive lipase: control of intracellular tri(di-)acylglycerol and cholesteryl ester hydrolysis. J. Lipid Res. 43, 1585-1594 (2002).

19. Osuga, J. et al. Targeted disruption of hormone-sensitive lipase results in male sterility and adipocyte hypertrophy, but not in obesity. Proc. Natl. Acad. Sci. U. S. A. 97, 787-792 (2000). 
20. Haemmerle, G. et al. Hormone-sensitive lipase deficiency in mice causes diglyceride accumulation in adipose tissue, muscle, and testis. J. Biol. Chem. 277, 4806-4815 (2002).

21. Park, S.-Y. et al. Hormone-sensitive lipase knockout mice have increased hepatic insulin sensitivity and are protected from short-term diet-induced insulin resistance in skeletal muscle and heart. Am. J. Physiol. Endocrinol. Metab. 289, E3039 (2005).

22. Harada, K. et al. Resistance to high-fat diet-induced obesity and altered expression of adipose-specific genes in HSL-deficient mice. Am. J. Physiol. Endocrinol. Metab. 285, E1182-1195 (2003).

23. Claus, T. H. et al. Specific inhibition of hormone-sensitive lipase improves lipid profile while reducing plasma glucose. J. Pharmacol. Exp. Ther. 315, 1396-1402 (2005).

24. Ebdrup, S., Refsgaard, H. H. F., Fledelius, C. \& Jacobsen, P. Synthesis and structure-activity relationship for a novel class of potent and selective carbamatebased inhibitors of hormone selective lipase with acute in vivo antilipolytic effects. $J$. Med. Chem. 50, 5449-5456 (2007).

25. Wei, E. et al. Loss of TGH/Ces3 in mice decreases blood lipids, improves glucose tolerance, and increases energy expenditure. Cell Metab. 11, 183-193 (2010).

26. Gilham, D. et al. Inhibitors of hepatic microsomal triacylglycerol hydrolase decrease very low density lipoprotein secretion. FASEB J. Off. Publ. Fed. Am. Soc. Exp. Biol. 17, 1685-1687 (2003).

27. Dominguez, E. et al. Integrated phenotypic and activity-based profiling links Ces3 to obesity and diabetes. Nat. Chem. Biol. 10, 113-121 (2014). 
28. Long, J. Z. \& Cravatt, B. F. The metabolic serine hydrolases and their functions in mammalian physiology and disease. Chem. Rev. 111, 6022-6063 (2011).

29. Yang, L. Y., Kuksis, A. \& Myher, J. J. Lipolysis of menhaden oil triacylglycerols and the corresponding fatty acid alkyl esters by pancreatic lipase in vitro: a reexamination. J. Lipid Res. 31, 137-147 (1990).

30. Whitcomb, D. C. \& Lowe, M. E. Human pancreatic digestive enzymes. Dig. Dis. Sci. 52, 1-17 (2007).

31. Hui, D. Y., Hayakawa, K. \& Oizumi, J. Lipoamidase activity in normal and mutagenized pancreatic cholesterol esterase (bile salt-stimulated lipase). Biochem. J. 291 ( Pt 1), 65-69 (1993).

32. Wang, C. S., Hartsuck, J. A. \& Downs, D. Kinetics of acylglycerol sequential hydrolysis by human milk bile salt activated lipase and effect of taurocholate as fatty acid acceptor. Biochemistry (Mosc.) 27, 4834-4840 (1988).

33. Mackay, K., Starr, J. R., Lawn, R. M. \& Ellsworth, J. L. Phosphatidylcholine hydrolysis is required for pancreatic cholesterol esterase- and phospholipase A2facilitated cholesterol uptake into intestinal Caco-2 cells. J. Biol. Chem. 272, 1338013389 (1997).

34. Aloulou, A. \& Carrière, F. Gastric lipase: an extremophilic interfacial enzyme with medical applications. Cell. Mol. Life Sci. CMLS 65, 851-854 (2008).

35. Lowe, M. E., Rosenblum, J. L. \& Strauss, A. W. Cloning and characterization of human pancreatic lipase cDNA. J. Biol. Chem. 264, 20042-20048 (1989).

36. Hui, D. Y. \& Howles, P. N. Carboxyl ester lipase: structure-function relationship and physiological role in lipoprotein metabolism and atherosclerosis. J. Lipid Res. 43, 2017-2030 (2002).

37. Bläckberg, L. \& Hernell, O. The bile-salt-stimulated lipase in human milk. Purification and characterization. Eur. J. Biochem. FEBS 116, 221-225 (1981). 
38. Rigtrup, K. M. \& Ong, D. E. A retinyl ester hydrolase activity intrinsic to the brush border membrane of rat small intestine. Biochemistry (Mosc.) 31, 2920-2926 (1992).

39. Moreau, H. et al. Immunocytolocalization of human gastric lipase in chief cells of the fundic mucosa. Histochemistry 91, 419-423 (1989).

40. Hernell, O. Human milk lipases. III. Physiological implications of the bile saltstimulated lipase. Eur. J. Clin. Invest. 5, 267-272 (1975).

41. Huggins, K. W., Camarota, L. M., Howles, P. N. \& Hui, D. Y. Pancreatic triglyceride lipase deficiency minimally affects dietary fat absorption but dramatically decreases dietary cholesterol absorption in mice. J. Biol. Chem. 278, 42899-42905 (2003).

42. Howles, P. N., Stemmerman, G. N., Fenoglio-Preiser, C. M. \& Hui, D. Y. Carboxyl ester lipase activity in milk prevents fat-derived intestinal injury in neonatal mice. Am. J. Physiol. 277, G653-661 (1999).

43. Kuusi, T., Kinnunen, P. K. \& Nikkilä, E. A. Hepatic endothelial lipase antiserum influences rat plasma low and high density lipoproteins in vivo. FEBS Lett. 104, 384388 (1979).

44. LaRosa, J. C., Levy, R. I., Windmueller, H. G. \& Fredrickson, D. S. Comparison of the triglyceride lipase of liver, adipose tissue, and postheparin plasma. J. Lipid Res. 13, 356-363 (1972).

45. Ehnholm, C., Shaw, W., Greten, H. \& Brown, W. V. Purification from human plasma of a heparin-released lipase with activity against triglyceride and phospholipids. J. Biol. Chem. 250, 6756-6761 (1975).

46. Connelly, P. W. The role of hepatic lipase in lipoprotein metabolism. Clin. Chim. Acta Int. J. Clin. Chem. 286, 243-255 (1999).

47. Jaye, M. et al. A novel endothelial-derived lipase that modulates HDL metabolism. Nat. Genet. 21, 424-428 (1999). 
48. Jin, W., Millar, J. S., Broedl, U., Glick, J. M. \& Rader, D. J. Inhibition of endothelial lipase causes increased HDL cholesterol levels in vivo. J. Clin. Invest. 111, 357-362 (2003).

49. Edmondson, A. C. et al. Loss-of-function variants in endothelial lipase are a cause of elevated HDL cholesterol in humans. J. Clin. Invest. 119, 1042-1050 (2009).

50. Sun, S. et al. Discovery of XEN445: a potent and selective endothelial lipase inhibitor raises plasma HDL-cholesterol concentration in mice. Bioorg. Med. Chem. 21, 7724-7734 (2013).

51. Mailly, F. et al. Familial lipoprotein lipase (LPL) deficiency: a catalogue of LPL gene mutations identified in 20 patients from the UK, Sweden, and Italy. Hum. Mutat. 10, 465-473 (1997).

52. Weinstock, P. H. et al. Severe hypertriglyceridemia, reduced high density lipoprotein, and neonatal death in lipoprotein lipase knockout mice. Mild hypertriglyceridemia with impaired very low density lipoprotein clearance in heterozygotes. J. Clin. Invest. 96, 2555-2568 (1995).

53. Goodman, K. B. et al. Discovery of potent, selective sulfonylfuran urea endothelial lipase inhibitors. Bioorg. Med. Chem. Lett. 19, 27-30 (2009).

54. Sugii, S., Reid, P. C., Ohgami, N., Du, H. \& Chang, T.-Y. Distinct endosomal compartments in early trafficking of low density lipoprotein-derived cholesterol. J. Biol. Chem. 278, 27180-27189 (2003).

55. Sheriff, S., Du, H. \& Grabowski, G. A. Characterization of lysosomal acid lipase by site-directed mutagenesis and heterologous expression. J. Biol. Chem. 270, 27766-27772 (1995).

56. Goldstein, J. L., Dana, S. E., Faust, J. R., Beaudet, A. L. \& Brown, M. S. Role of lysosomal acid lipase in the metabolism of plasma low density lipoprotein. 
Observations in cultured fibroblasts from a patient with cholesteryl ester storage disease. J. Biol. Chem. 250, 8487-8495 (1975).

57. Du, H. et al. Lysosomal acid lipase-deficient mice: depletion of white and brown fat, severe hepatosplenomegaly, and shortened life span. J. Lipid Res. 42, 489-500 (2001).

58. Du, H., Duanmu, M., Witte, D. \& Grabowski, G. A. Targeted disruption of the mouse lysosomal acid lipase gene: long-term survival with massive cholesteryl ester and triglyceride storage. Hum. Mol. Genet. 7, 1347-1354 (1998).

59. Aslanidis, C. et al. Genetic and biochemical evidence that CESD and Wolman disease are distinguished by residual lysosomal acid lipase activity. Genomics 33, 85-93 (1996).

60. Anderson, R. A., Byrum, R. S., Coates, P. M. \& Sando, G. N. Mutations at the lysosomal acid cholesteryl ester hydrolase gene locus in Wolman disease. Proc. Natl. Acad. Sci. U. S. A. 91, 2718-2722 (1994).

61. Hoeg, J. M., Demosky, S. J., Pescovitz, O. H. \& Brewer, H. B. Cholesteryl ester storage disease and Wolman disease: phenotypic variants of lysosomal acid cholesteryl ester hydrolase deficiency. Am. J. Hum. Genet. 36, 1190-1203 (1984).

62. Rosenbaum, A. I. et al. Thiadiazole carbamates: potent inhibitors of lysosomal acid lipase and potential Niemann-Pick type C disease therapeutics. J. Med. Chem. $53,5281-5289$ (2010).

63. Long, J. Z., Nomura, D. K. \& Cravatt, B. F. Characterization of monoacylglycerol lipase inhibition reveals differences in central and peripheral endocannabinoid metabolism. Chem. Biol. 16, 744-753 (2009).

64. Kohnz, R. A. \& Nomura, D. K. Chemical approaches to therapeutically target the metabolism and signaling of the endocannabinoid 2-AG and eicosanoids. Chem. Soc. Rev. 43, 6859-6869 (2014). 
65. Mulvihill, M. M. \& Nomura, D. K. Therapeutic potential of monoacylglycerol lipase inhibitors. Life Sci. 92, 492-497 (2013).

66. Chang, J. W. et al. Highly selective inhibitors of monoacylglycerol lipase bearing a reactive group that is bioisosteric with endocannabinoid substrates. Chem. Biol. 19, 579-588 (2012).

67. Chang, J. W., Cognetta, A. B., 3rd, Niphakis, M. J. \& Cravatt, B. F. Proteomewide reactivity profiling identifies diverse carbamate chemotypes tuned for serine hydrolase inhibition. ACS Chem. Biol. 8, 1590-1599 (2013).

68. Long, J. Z. et al. Selective blockade of 2-arachidonoylglycerol hydrolysis produces cannabinoid behavioral effects. Nat. Chem. Biol. 5, 37-44 (2009).

69. Nomura, D. K. et al. Endocannabinoid hydrolysis generates brain prostaglandins that promote neuroinflammation. Science 334, 809-813 (2011).

70. Long, J. Z., Nomura, D. K. \& Cravatt, B. F. Characterization of monoacylglycerol lipase inhibition reveals differences in central and peripheral endocannabinoid metabolism. Chem. Biol. 16, 744-753 (2009).

71. Schlosburg, J. E. et al. Chronic monoacylglycerol lipase blockade causes functional antagonism of the endocannabinoid system. Nat. Neurosci. 13, 1113-1119 (2010).

72. Fernández-Suárez, D. et al. Monoacylglycerol lipase inhibitor JZL184 is neuroprotective and alters glial cell phenotype in the chronic MPTP mouse model. Neurobiol. Aging 35, 2603-2616 (2014).

73. Piro, J. R. et al. A dysregulated endocannabinoid-eicosanoid network supports pathogenesis in a mouse model of Alzheimer's disease. Cell Rep. 1, 617-623 (2012).

74. Chen, R. et al. Monoacylglycerol lipase is a therapeutic target for Alzheimer's disease. Cell Rep. 2, 1329-1339 (2012). 
75. Sanchez-Alavez, M. et al. Monoacylglycerol lipase regulates fever response.

PloS One 10, e0134437 (2015).

76. Cao, Z. et al. Monoacylglycerol lipase controls endocannabinoid and eicosanoid signaling and hepatic injury in mice. Gastroenterology 144, 808-817.e15 (2013).

77. Taschler, U. et al. Monoglyceride lipase deficiency in mice impairs lipolysis and attenuates diet-induced insulin resistance. J. Biol. Chem. 286, 17467-17477 (2011).

78. Viader, A. et al. Metabolic Interplay between Astrocytes and Neurons Regulates Endocannabinoid Action. Cell Rep. 12, 798-808 (2015).

79. Nomura, D. K. et al. Monoacylglycerol lipase regulates a fatty acid network that promotes cancer pathogenesis. Cell 140, 49-61 (2010).

80. Nomura, D. K. et al. Monoacylglycerol lipase exerts dual control over endocannabinoid and fatty acid pathways to support prostate cancer. Chem. Biol. 18, 846-856 (2011).

81. Abide Therapeutics Announces Clinical Study of ABX-1431. ABIDE THERAPEUTICS at <http://abidetx.com/abide-therapeutics-announces-first-subjectdosed-in-first-in-human-clinical-study-of-abx-1431-an-investigationalendocannabinoid-system-modulator/>

82. Bisogno, T. et al. Cloning of the first sn1-DAG lipases points to the spatial and temporal regulation of endocannabinoid signaling in the brain. J. Cell Biol. 163, 463468 (2003).

83. Gao, Y. et al. Loss of retrograde endocannabinoid signaling and reduced adult neurogenesis in diacylglycerol lipase knock-out mice. J. Neurosci. Off. J. Soc. Neurosci. 30, 2017-2024 (2010).

84. Tanimura, A. et al. The endocannabinoid 2-arachidonoylglycerol produced by diacylglycerol lipase alpha mediates retrograde suppression of synaptic transmission. Neuron 65, 320-327 (2010). 
85. Jenniches, I. et al. Anxiety, stress, and fear response in mice with reduced endocannabinoid levels. Biol. Psychiatry (2015). doi:10.1016/j.biopsych.2015.03.033

86. Hsu, K.-L. et al. DAGL $\beta$ inhibition perturbs a lipid network involved in macrophage inflammatory responses. Nat. Chem. Biol. 8, 999-1007 (2012).

87. Baggelaar, M. P. et al. Highly selective, reversible inhibitor identified by comparative chemoproteomics modulates diacylglycerol lipase activity in neurons. $J$. Am. Chem. Soc. 137, 8851-8857 (2015).

88. Nomura, D. K. et al. Serine hydrolase KIAA1363: toxicological and structural features with emphasis on organophosphate interactions. Chem. Res. Toxicol. 19, 1142-1150 (2006).

89. Chiang, K. P., Niessen, S., Saghatelian, A. \& Cravatt, B. F. An enzyme that regulates ether lipid signaling pathways in cancer annotated by multidimensional profiling. Chem. Biol. 13, 1041-1050 (2006).

90. Nomura, D. K. et al. Dual roles of brain serine hydrolase KIAA1363 in ether lipid metabolism and organophosphate detoxification. Toxicol. Appl. Pharmacol. 228, 4248 (2008).

91. Chang, J. W., Nomura, D. K. \& Cravatt, B. F. A potent and selective inhibitor of KIAA1363/AADACL1 that impairs prostate cancer pathogenesis. Chem. Biol. 18, 476-484 (2011).

92. Holly, S. P. et al. Chemoproteomic discovery of AADACL1 as a regulator of human platelet activation. Chem. Biol. 20, 1125-1134 (2013).

93. Nomura, D. K. et al. Activation of the endocannabinoid system by organophosphorus nerve agents. Nat. Chem. Biol. 4, 373-378 (2008).

94. Cravatt, B. F. et al. Molecular characterization of an enzyme that degrades neuromodulatory fatty-acid amides. Nature 384, 83-87 (1996). 
95. Ahn, K., McKinney, M. K. \& Cravatt, B. F. Enzymatic pathways that regulate endocannabinoid signaling in the nervous system. Chem. Rev. 108, 1687-1707 (2008).

96. Saghatelian, A., McKinney, M. K., Bandell, M., Patapoutian, A. \& Cravatt, B. F. A FAAH-regulated class of $\mathrm{N}$-acyl taurines that activates TRP ion channels.

Biochemistry (Mosc.) 45, 9007-9015 (2006).

97. Long, J. Z., LaCava, M., Jin, X. \& Cravatt, B. F. An anatomical and temporal portrait of physiological substrates for fatty acid amide hydrolase. J. Lipid Res. 52, 337-344 (2011).

98. Cravatt, B. F. et al. Supersensitivity to anandamide and enhanced endogenous cannabinoid signaling in mice lacking fatty acid amide hydrolase. Proc. Natl. Acad. Sci. U. S. A. 98, 9371-9376 (2001).

99. Ahn, K. et al. Discovery and characterization of a highly selective FAAH inhibitor that reduces inflammatory pain. Chem. Biol. 16, 411-420 (2009).

100. Ahn, K. et al. Mechanistic and pharmacological characterization of PF-04457845: a highly potent and selective fatty acid amide hydrolase inhibitor that reduces inflammatory and noninflammatory pain. J. Pharmacol. Exp. Ther. 338, 114-124 (2011).

101. Kathuria, S. et al. Modulation of anxiety through blockade of anandamide hydrolysis. Nat. Med. 9, 76-81 (2003).

102. Kinsey, S. G. et al. Blockade of endocannabinoid-degrading enzymes attenuates neuropathic pain. J. Pharmacol. Exp. Ther. 330, 902-910 (2009).

103. Naidu, P. S. et al. Evaluation of fatty acid amide hydrolase inhibition in murine models of emotionality. Psychopharmacology (Berl.) 192, 61-70 (2007).

104. Kinsey, S. G., Long, J. Z., Cravatt, B. F. \& Lichtman, A. H. Fatty acid amide hydrolase and monoacylglycerol lipase inhibitors produce anti-allodynic effects in 
mice through distinct cannabinoid receptor mechanisms. J. Pain Off. J. Am. Pain Soc. $11,1420-1428$ (2010).

105. Cravatt, B. F. et al. Functional disassociation of the central and peripheral fatty acid amide signaling systems. Proc. Natl. Acad. Sci. U. S. A. 101, 10821-10826 (2004).

106. Kinsey, S. G., O’Neal, S. T., Long, J. Z., Cravatt, B. F. \& Lichtman, A. H. Inhibition of endocannabinoid catabolic enzymes elicits anxiolytic-like effects in the marble burying assay. Pharmacol. Biochem. Behav. 98, 21-27 (2011).

107. Booker, L. et al. The fatty acid amide hydrolase (FAAH) inhibitor PF-3845 acts in the nervous system to reverse LPS-induced tactile allodynia in mice. Br. J. Pharmacol. 165, 2485-2496 (2012).

108. Lichtman, A. H. et al. Reversible inhibitors of fatty acid amide hydrolase that promote analgesia: evidence for an unprecedented combination of potency and selectivity. J. Pharmacol. Exp. Ther. 311, 441-448 (2004).

109. Lodola, A., Castelli, R., Mor, M. \& Rivara, S. Fatty acid amide hydrolase inhibitors: a patent review (2009 - 2014). Expert Opin. Ther. Pat. 25, 1247-1266 (2015).

110. Dennis, E. A., Cao, J., Hsu, Y.-H., Magrioti, V. \& Kokotos, G. Phospholipase A2 enzymes: physical structure, biological function, disease implication, chemical inhibition, and therapeutic intervention. Chem. Rev. 111, 6130-6185 (2011).

111. Diez, E., Louis-Flamberg, P., Hall, R. H. \& Mayer, R. J. Substrate specificities and properties of human phospholipases A2 in a mixed vesicle model. J. Biol. Chem. 267, 18342-18348 (1992).

112. Bonventre, J. V. et al. Reduced fertility and postischaemic brain injury in mice deficient in cytosolic phospholipase A2. Nature 390, 622-625 (1997). 
113. Marusic, S. et al. Cytosolic phospholipase A2 alpha-deficient mice are resistant to experimental autoimmune encephalomyelitis. J. Exp. Med. 202, 841-851 (2005).

114. Ishihara, K. et al. Group IVA phospholipase A2 participates in the progression of hepatic fibrosis. FASEB J. Off. Publ. Fed. Am. Soc. Exp. Biol. 26, 4111-4121 (2012).

115. Uozumi, N. et al. Role of cytosolic phospholipase A2 in allergic response and parturition. Nature 390, 618-622 (1997).

116. Hsu, K.-L. et al. DAGL $\beta$ inhibition perturbs a lipid network involved in macrophage inflammatory responses. Nat. Chem. Biol. 8, 999-1007 (2012).

117. Seno, K. et al. Pyrrolidine inhibitors of human cytosolic phospholipase A2. Part 2: synthesis of potent and crystallized 4-triphenylmethylthio derivative 'pyrrophenone'. Bioorg. Med. Chem. Lett. 11, 587-590 (2001).

118. Lee, K. L. et al. Discovery of Ecopladib, an indole inhibitor of cytosolic phospholipase A2alpha. J. Med. Chem. 50, 1380-1400 (2007).

119. Lio, Y. C. \& Dennis, E. A. Interfacial activation, lysophospholipase and transacylase activity of group VI Ca2+-independent phospholipase A2. Biochim. Biophys. Acta 1392, 320-332 (1998).

120. Tang, J. et al. A novel cytosolic calcium-independent phospholipase A2 contains eight ankyrin motifs. J. Biol. Chem. 272, 8567-8575 (1997).

121. Bao, S. et al. Male mice that do not express group VIA phospholipase A2 produce spermatozoa with impaired motility and have greatly reduced fertility. J. Biol. Chem. 279, 38194-38200 (2004).

122. Shinzawa, K. et al. Neuroaxonal dystrophy caused by group VIA phospholipase A2 deficiency in mice: a model of human neurodegenerative disease. J. Neurosci. Off. J. Soc. Neurosci. 28, 2212-2220 (2008).

123. Morgan, N. V. et al. PLA2G6, encoding a phospholipase A2, is mutated in neurodegenerative disorders with high brain iron. Nat. Genet. 38, 752-754 (2006). 
124. Khateeb, S. et al. PLA2G6 mutation underlies infantile neuroaxonal dystrophy. Am. J. Hum. Genet. 79, 942-948 (2006).

125. Tjoelker, L. W. et al. Anti-inflammatory properties of a platelet-activating factor acetylhydrolase. Nature 374, 549-553 (1995).

126. Stafforini, D. M., Elstad, M. R., McIntyre, T. M., Zimmerman, G. A. \& Prescott, S. M. Human macrophages secret platelet-activating factor acetylhydrolase. J. Biol. Chem. 265, 9682-9687 (1990).

127. Stafforini, D. M., McIntyre, T. M., Carter, M. E. \& Prescott, S. M. Human plasma platelet-activating factor acetylhydrolase. Association with lipoprotein particles and role in the degradation of platelet-activating factor. J. Biol. Chem. 262, 4215-4222 (1987).

128. Stremler, K. E., Stafforini, D. M., Prescott, S. M. \& Mclntyre, T. M. Human plasma platelet-activating factor acetylhydrolase. Oxidatively fragmented phospholipids as substrates. J. Biol. Chem. 266, 11095-11103 (1991).

129. Stremler, K. E., Stafforini, D. M., Prescott, S. M., Zimmerman, G. A. \& McIntyre, T. M. An oxidized derivative of phosphatidylcholine is a substrate for the plateletactivating factor acetylhydrolase from human plasma. J. Biol. Chem. 264, 5331-5334 (1989).

130. Karasawa, K., Harada, A., Satoh, N., Inoue, K. \& Setaka, M. Plasma platelet activating factor-acetylhydrolase (PAF-AH). Prog. Lipid Res. 42, 93-114 (2003).

131. Nagano, J. M. G. et al. Selective inhibitors and tailored activity probes for lipoprotein-associated phospholipase A(2). Bioorg. Med. Chem. Lett. 23, 839-843 (2013).

132. Tawakol, A. et al. Effect of treatment for 12 weeks with rilapladib, a lipoproteinassociated phospholipase A2 inhibitor, on arterial inflammation as assessed with $18 \mathrm{~F}$ - 
fluorodeoxyglucose-positron emission tomography imaging. J. Am. Coll. Cardiol. 63, 86-88 (2014).

133. Serruys, P. W. et al. Effects of the direct lipoprotein-associated phospholipase A(2) inhibitor darapladib on human coronary atherosclerotic plaque. Circulation 118, 1172-1182 (2008).

134. Wilensky, R. L. et al. Inhibition of lipoprotein-associated phospholipase A2 reduces complex coronary atherosclerotic plaque development. Nat. Med. 14, 10591066 (2008).

135. Acharya, N. K. et al. Diabetes and hypercholesterolemia increase blood-brain barrier permeability and brain amyloid deposition: beneficial effects of the LpPLA2 inhibitor darapladib. J. Alzheimers Dis. JAD 35, 179-198 (2013).

136. Moser, M. et al. Cloning and expression of the murine sws/NTE gene. Mech. Dev. 90, 279-282 (2000).

137. Akassoglou, K. et al. Brain-specific deletion of neuropathy target esterase/swisscheese results in neurodegeneration. Proc. Natl. Acad. Sci. U. S. A. 101, 5075-5080 (2004).

138. van Tienhoven, M., Atkins, J., Li, Y. \& Glynn, P. Human neuropathy target esterase catalyzes hydrolysis of membrane lipids. J. Biol. Chem. 277, 20942-20948 (2002).

139. Quistad, G. B., Barlow, C., Winrow, C. J., Sparks, S. E. \& Casida, J. E. Evidence that mouse brain neuropathy target esterase is a lysophospholipase. Proc. Natl. Acad. Sci. U. S. A. 100, 7983-7987 (2003).

140. Bae, K. et al. Platelet-activating factor (PAF)-dependent transacetylase and its relationship with PAF acetylhydrolases. J. Biol. Chem. 275, 26704-26709 (2000).

141. Hattori, K. et al. Purification and characterization of platelet-activating factor acetylhydrolase II from bovine liver cytosol. J. Biol. Chem. 270, 22308-22313 (1995). 
142. Kono, N. et al. Protection against oxidative stress-induced hepatic injury by intracellular type II platelet-activating factor acetylhydrolase by metabolism of oxidized phospholipids in vivo. J. Biol. Chem. 283, 1628-1636 (2008).

143. Adibekian, A. et al. Click-generated triazole ureas as ultrapotent in vivo-active serine hydrolase inhibitors. Nat. Chem. Biol. 7, 469-478 (2011).

144. Hattori, M. et al. Cloning and expression of a cDNA encoding the beta-subunit (30-kDa subunit) of bovine brain platelet-activating factor acetylhydrolase. J. Biol. Chem. 270, 31345-31352 (1995).

145. Hattori, M., Adachi, H., Tsujimoto, M., Arai, H. \& Inoue, K. The catalytic subunit of bovine brain platelet-activating factor acetylhydrolase is a novel type of serine esterase. J. Biol. Chem. 269, 23150-23155 (1994).

146. Koizumi, H. et al. Targeted disruption of intracellular type I platelet activating factor-acetylhydrolase catalytic subunits causes severe impairment in spermatogenesis. J. Biol. Chem. 278, 12489-12494 (2003).

147. Hirotsune, S. et al. Graded reduction of Pafah1b1 (Lis1) activity results in neuronal migration defects and early embryonic lethality. Nat. Genet. 19, 333-339 (1998).

148. Nilsson, R. et al. Metabolic enzyme expression highlights a key role for MTHFD2 and the mitochondrial folate pathway in cancer. Nat. Commun. 5, 3128 (2014).

149. Mulvihill, M. M. et al. Metabolic Profiling Reveals PAFAH1B3 as a Critical Driver of Breast Cancer Pathogenicity. Chem. Biol. 21, 831-840 (2014).

150. Chang, J. W. et al. A selective inhibitor of platelet-activating factor acetylhydrolases $1 \mathrm{~b} 2$ and $1 \mathrm{~b} 3$ that impairs cancer cell survival. ACS Chem. Biol. 10, 925-932 (2015). 
151. Lee, H.-C., Simon, G. M. \& Cravatt, B. F. ABHD4 regulates multiple classes of Nacyl phospholipids in the mammalian central nervous system. Biochemistry (Mosc.)

54, 2539-2549 (2015).

152. Kamat, S. S. et al. Immunomodulatory lysophosphatidylserines are regulated by ABHD16A and ABHD12 interplay. Nat. Chem. Biol. 11, 164-171 (2015).

153. Nomura, D. K. et al. A brain detoxifying enzyme for organophosphorus nerve poisons. Proc. Natl. Acad. Sci. U. S. A. 102, 6195-6200 (2005).

154. Nomura, D. K. et al. Activation of the endocannabinoid system by organophosphorus nerve agents. Nat. Chem. Biol. 4, 373-378 (2008).

155. Medina-Cleghorn, D., Heslin, A., Morris, P. J., Mulvihill, M. M. \& Nomura, D. K. Multidimensional Profiling Platforms Reveal Metabolic Dysregulation Caused by Organophosphorus Pesticides. ACS Chem. Biol. 9, 423-432 (2014).

156. Morris, P. J. et al. Organophosphorus flame retardants inhibit specific liver carboxylesterases and cause serum hypertriglyceridemia. ACS Chem. Biol. 9, 10971103 (2014).

157. Casida, J. E., Eto, M. \& Baron, R. L. Biological activity of a trio-cresyl phosphate metabolite. Nature 191, 1396-1397 (1961).

158. Long, J. Z. et al. Dual blockade of FAAH and MAGL identifies behavioral processes regulated by endocannabinoid crosstalk in vivo. Proc. Natl. Acad. Sci. U. S. A. 106, 20270-20275 (2009). 
Figure Legends

Figure 1. Neutral lipid lipase pathways (A) and inhibitors (B)

Figure 2. N-Acylethanolamine pathway (A) and FAAH inhibitors (B)

Figure 3. Phospholipase pathways (A) and inhibitors (B)

Figure 4. Environmental agents that inhibit lipases 


\section{neutral lipid lipase pathways}<smiles>[R]C(=O)OCC(COC([R])=O)OC(=O)O</smiles>

triglycerides

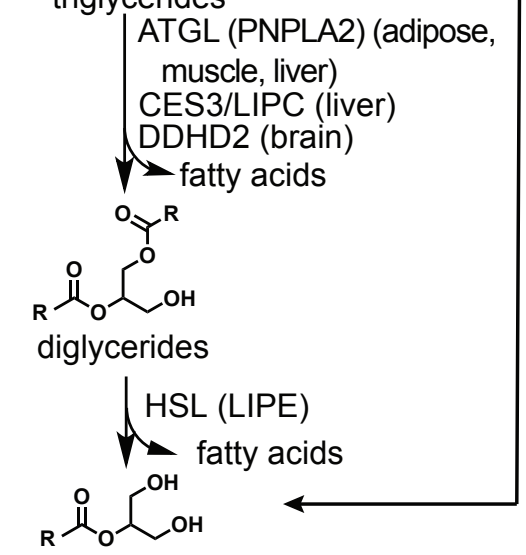

monoglycerides

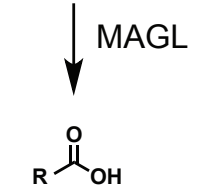

fatty acids

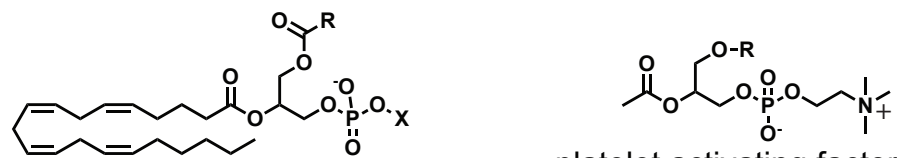

PNLIP phospholipid
CEL

LIPF

(stomach/ intestinal/

lumen)

LPL

(capillary

endothelia)

LIPA

(lysosomes)

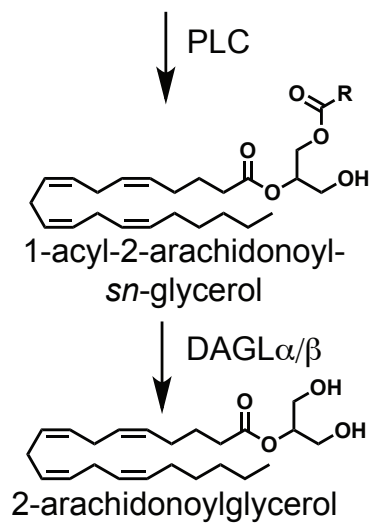

(endocannabinoid)

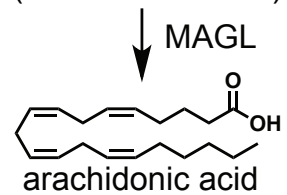

platelet activating factor (PAF)

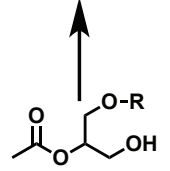

2-acetyl monoalkylglycerol ether

(2-acetyl MAGE)

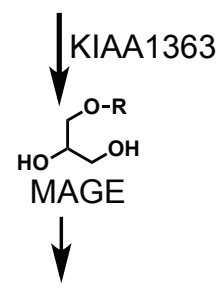

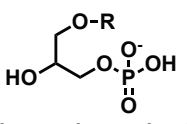

alkyl lysophosphatidic acid

\section{neutral lipid lipase inhibitors}<smiles>CN(C)C(=O)Nc1cccc(-c2ccc(N(C)C)cc2)c1</smiles>

Atglstatin

ATGL inhibitor<smiles>CCCCCCCCCCC1CC(CC(=O)NC(CC(C)C)C(=O)NC=O)OC(CCCCCC)OC1=O</smiles>
non-specific lipase inhibitor (e.g. PNLIP, LIPF)

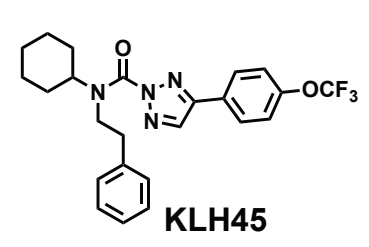

DDHD2 inhibitor<smiles>Cc1cccc(NC(=O)C(Nc2cccs2)C(=O)C(F)(F)F)c1</smiles>

GR148672X<smiles>CCOC(=O)c1ccc(-c2ccc(OC(=O)N(C)Cc3cccc(-c4cc[nH]c4)c3)cc2)cc1</smiles>

WWL113

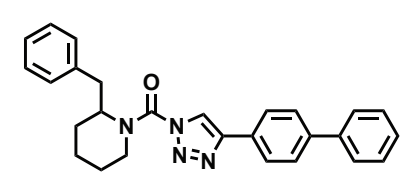

KT109

DAGL $\beta$ inhibitor

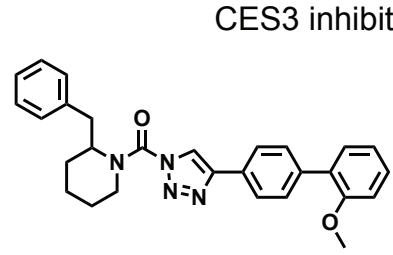

KT172 DAGL $\beta$ inhibitor DAGL inhibitors

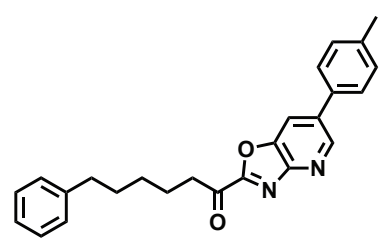

LEI105
DAGL $\alpha / \beta$ inhibitor

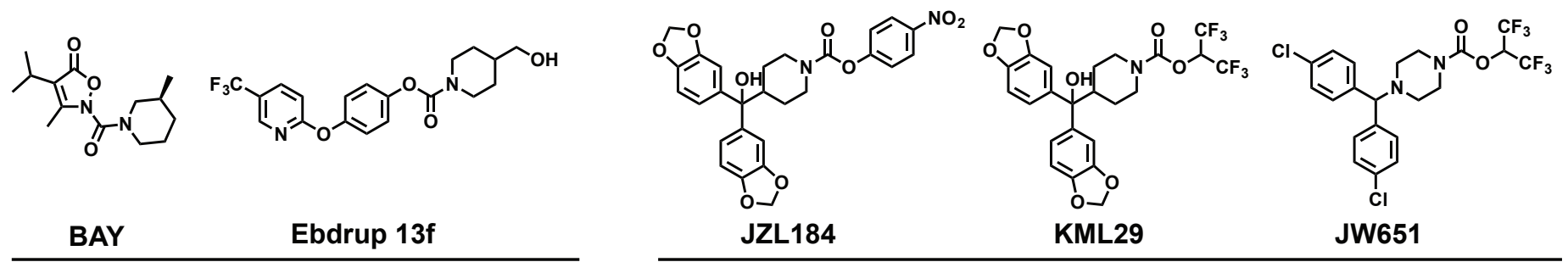

HSL inhibitors

MAGL inhibitors<smiles>O=C(O)c1cc(C(F)(F)F)ccc1N1CCC(OCC2CCCN2)C1</smiles><smiles>CC(C)c1ccccc1OC(=O)NCCc1ccc2ccccc2c1</smiles> 


\section{Figure 2}

A N-acylethanolamine pathway

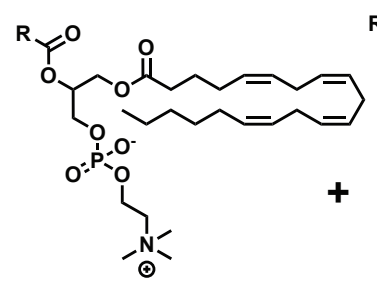

phosphatidylcholine phosphatidylethanolamine

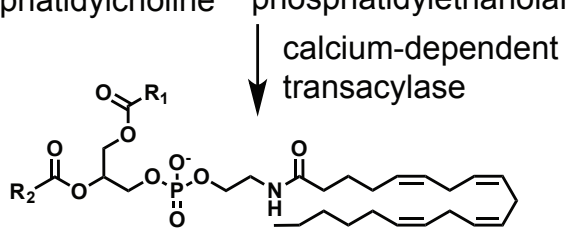

$\mathrm{N}$-acyl phosphatidylethanolamine

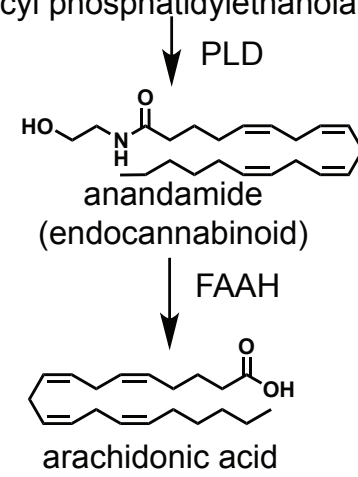

B FAAH inhibitors

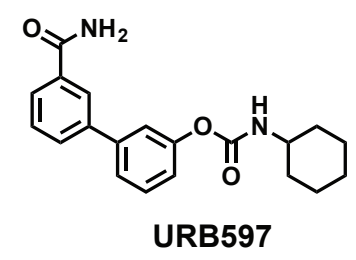<smiles></smiles>

OL-135<smiles>O=C(Nc1cccnc1)N1CCC(Cc2cccc(Oc3ccc(C(F)(F)F)cn3)c2)CC1</smiles>

PF-3845

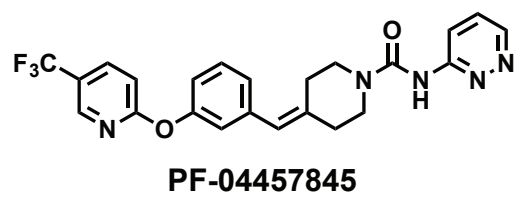




\section{Figure 3}

A

general phospholipase activity

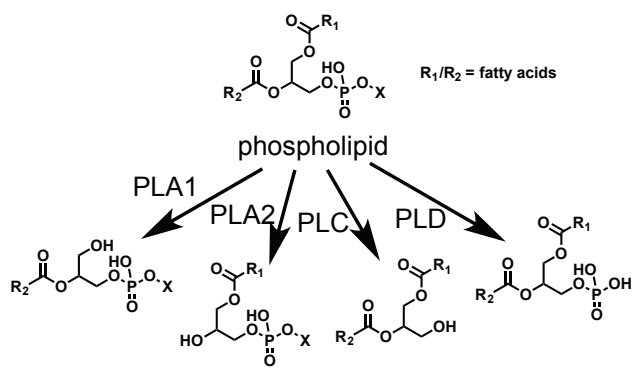

phosphatidylserine metabolism

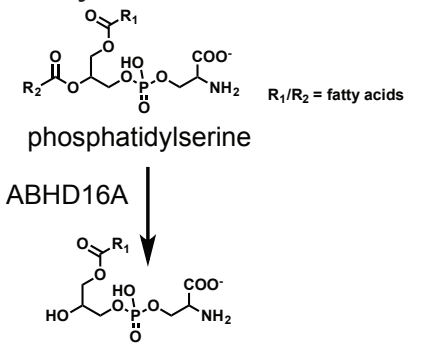

lysophosphatidylserine

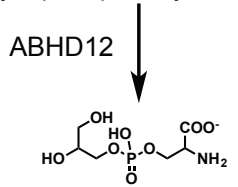

glycerophosphoserine

\section{phospholipase pathways}

PLA2G4A regulation of arachidonic acid and eicosanoids

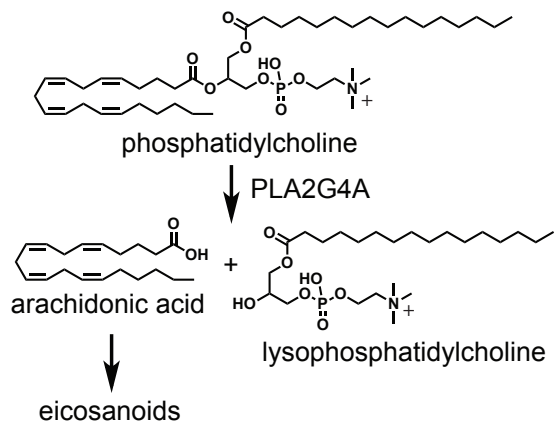

PLA2G7 and PAFAH2 act on

PAF and oxidized phospholipids

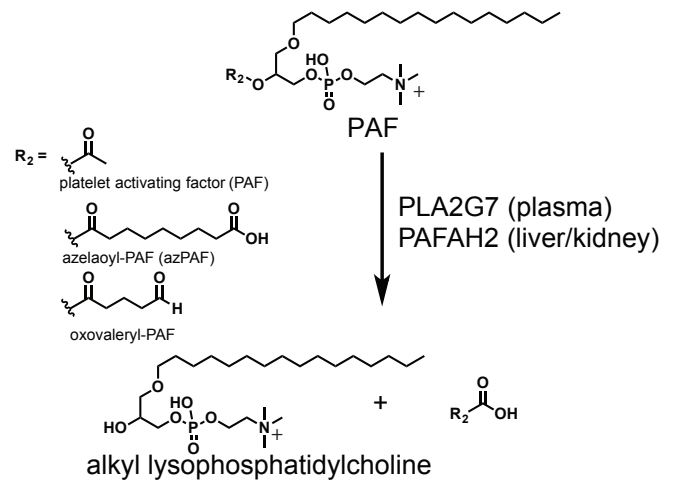

ABHD3 acts on medium chain and oxidized phospholipids

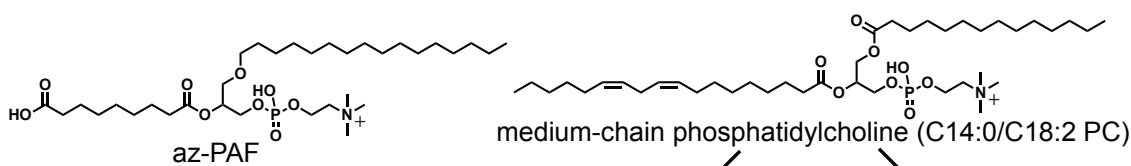

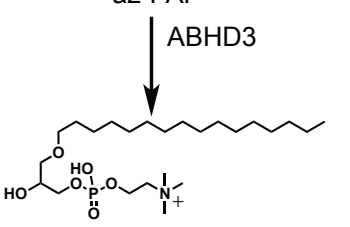

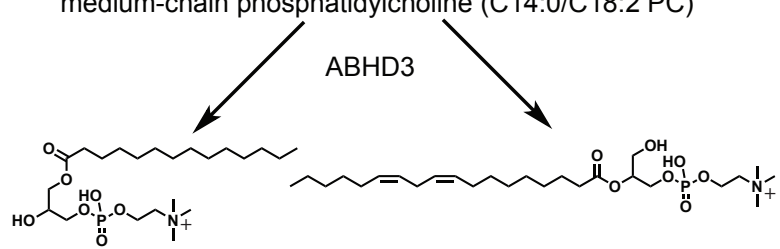

B

\section{phospholipase inhibitors}

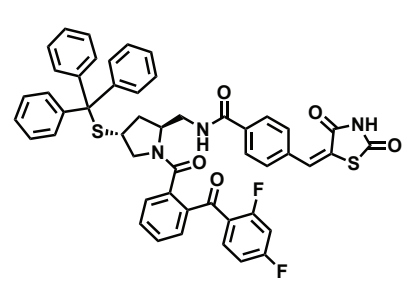

pyrrophenone

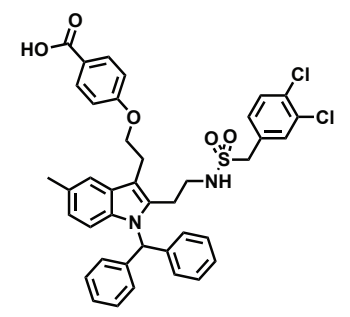

Ecopladib

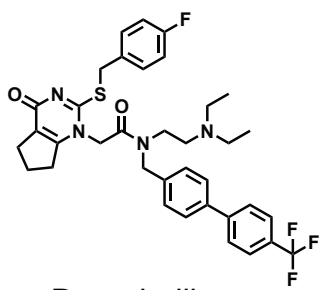

Darapladib

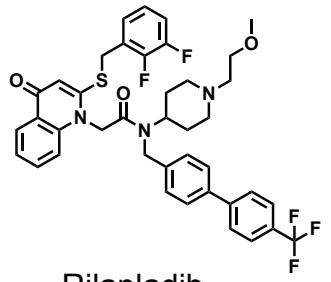

Rilapladib

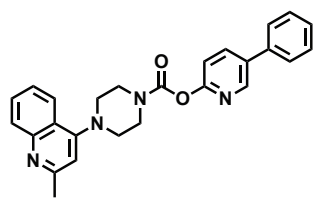

JMN21

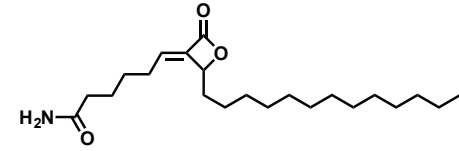

KC01

ABHD16A inhibitor<smiles></smiles>

AA39-2

PAFAH2 inhibitor 


\section{Figure 4}

\section{A organophosphate-induced delayed neuropathy from PNPLA6/NTE inhibition and aging}

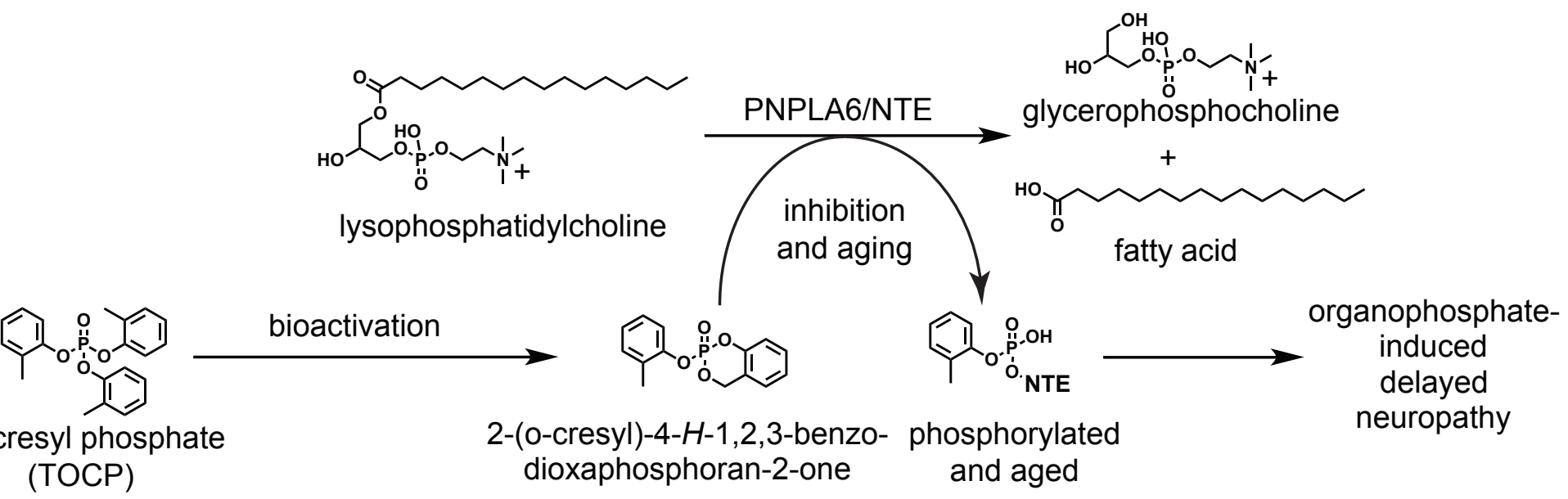

B

pesticide-induced cannabinoid behaviors from MAGL and FAAH inhibition

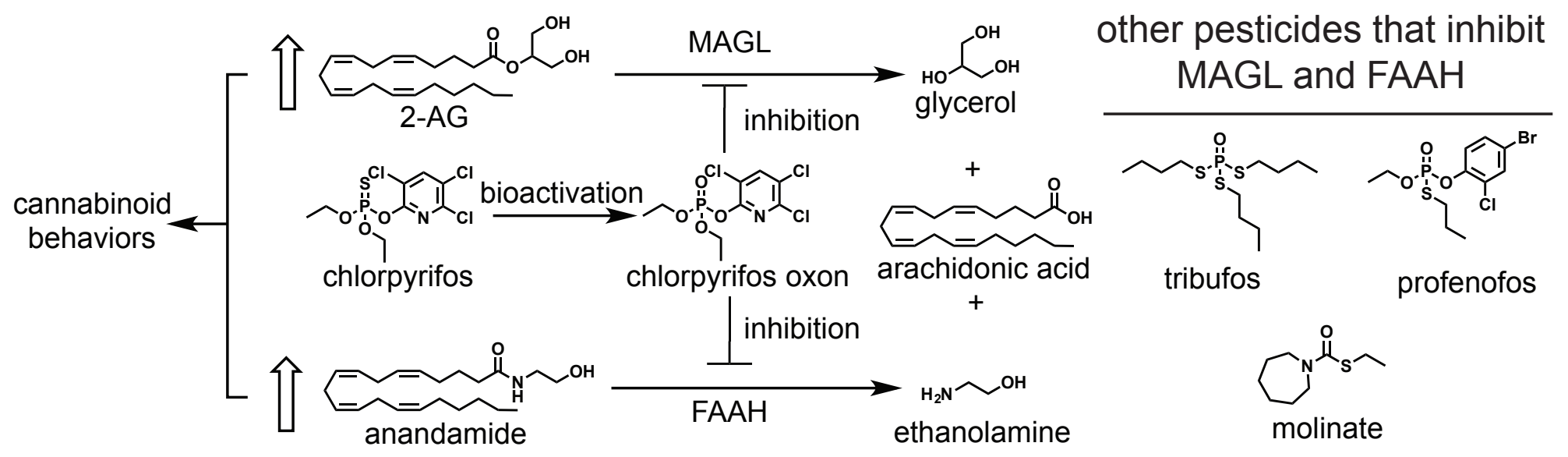

